\title{
Cavum Septum Pellucidum: Hallazgo Mediante Disección Neuroanatómica
}

\author{
Cavum Septum Pellucidum: Finding from Neuroanatomical Dissection
}

\author{
*,*** Jorge Eduardo Duque Parra
}

\begin{abstract}
DUQUE PARRA, J. E. Cavum septum pellucidum: hallazgo mediante disección neuroanatómica. Int. J. Morphol., 30(4):1508-1511, 2012.
RESUMEN: Se reporta una marcada cavitación a nivel del septum pellucidum, un gran cavum septum pellucidum de una persona adulta, en el espacio reconocido generalmente como cerrado del sistema nervioso central, que se hace evidente como tal, en ciertas fases de la vida intrauterina. Esta cavitación está ausente en el adulto sano, pero en algunas personas esquizofrénicas y con demencia pugilística, dicha cavidad, puede considerarse como un hallazgo de relativa frecuencia. Este hallazgo se encontró aleatoriamente en uno de cuarenta y dos encéfalos de adultos, valorados superficialmente desde la anatomía macroscópica como normales. Dicho hallazgo posiblemente se asocie con desórdenes conductuales, en vista que dicho encéfalo correspondía a un individuo proveniente de un hospital psiquiátrico.
\end{abstract}

PALABRAS CLAVE: Cavum septum pellucidum; Neuroanatomía; Disección.

\section{INTRODUCCIÓN}

El septum pellucidum es un tabique transparente (Testut \& Latarjet, 1978; Duque Parra, 2001, 2004), delgado, vertical que consta de dos láminas (Testut \& Latarjet; Duque Parra, 2001, 2004; Pearce, 2008; Ranson \& Clark, 1959; Tubbs et al., 2011) separadas por un estrecho intervalo llamado cavidad del septum pellucidum (Ranson \& Clark; Duque Parra, 2001). Este espacio cerrado (Testut \& Latarjet; Kuhlenbeck, 1973) es de variadas dimensiones y no comunica con los ventrículos laterales (Testeut \& Latarjet; Clemente, 1985; Kuhlenbeck; Kiernan, 2009; Aldur et al., 1997) o con el espacio subaracnoideo (Kiernan, Duque Parra, 2002). Su forma es trigonal de base anterior y vértice posterior, sus láminas se unen cranealmente a la superficie cóncava inferior del tronco, rodilla y pico del cuerpo calloso. Caudal y dorsalmente se une a la porción anterior del fórnix, ventral y rostralmente a la superficie superior del cuerpo calloso (Clemente; Pearce). Las láminas están formando las mitades derecha e izquierda del septum pellucidum. Durante el neurodesarrollo, la lámina terminal que conecta los dos hemisferios cerebrales, sirve de puente para fibras que cruzan de un hemisferio a otro. La porción de la lámina terminal que entra en la formación del septum pellucidum llega a ahuecarse como resultado del estiramiento al cual es sometido y la cavidad resultante es la cavidad del septum pellucidum (Ranson \& Clark), por lo tanto es una porción de la fisura interhemisférica, que se ha aislado en el transcurso del neurodesarrollo, así es que no tiene ninguna relación con la cavidad central del neuroeje embrionario (Testut \& Latarjet), mas en el feto, la cavidad del septum pellucidum es amplia y se puede visualizar mediante técnicas no invasivas que utilizan ultrasonidos (Martin, 1998) o imágenes de resonancia magnética (Tubbs et al.), o de otro tipo, aunque no hay reportes de abordajes neuroanatómicos.

Esta cavidad mal o impropiamente denominada quinto ventrículo o ventrículo del tabique (Testut \& Latarjet; Ranson \& Clark; Afifi \& Bergman, 1999), indica por su nombre la posición exacta y no prejuzga nada de su naturaleza (Testut \& Latarjet), ya que no hace parte del sistema ventricular (Ranson \& Clark; Duque Parra, 2004) como algunos aún sostienen. Por término medio, mide $3 \mathrm{~cm}$ de largo, su ancho en su parte superior es de 2 a $3 \mathrm{~mm}$, generalmente terminando hacia atrás en la parte media del cuerpo calloso (Testut \& Latarjet). El cavum septum pellucidum cuando es amplio, es comúnmente descrito como un hallazgo asociado con enfermedades y anormalidades (Bodensteiner \& Shaefer, 1990). Clínicamente se ha relacionado como una gran cavidad en el encéfalo de boxeadores profesionales (Kiernan; Martin; Bogfdanoff \& Natter, 1990, Pearce) y futbolistas (Grossman \& Yousem, 2007) quienes han recibido traumas a repetición, en los que es mas frecuente esta cavidad que en personas que no presentaron tal condición (Grossman \& Yousem; Filipovic

\footnotetext{
* Grupo Neurociencia de Caldas (Colciencias). Departamento de Ciencias Básicas, Programa de Medicina, Universidad de Caldas, Manizales, Colombia. **Grupo Neuroaprendizaje de la UAM (Colciencias). Departamento de Ciencias Básicas Biológicas, Universidad Autónoma de Manizales, Manizales, Colombia.
} 
et al., 1996) y que sufren demencia pugilística (Martin).

Se ha atribuido esta cavidad a los traumatismos cefálicos repetidos con desgarro de las paredes septales y disección secundaria de los tabiques por el líquido cerebro epinal con formación de la cavidad (Grossman \& Yousem). También se ha relacionado con displasia septo óptica (Pearce), hipoplasia del nervio óptico (Zimmermann-Paiz \& Fang Sung, 2009) y quistes (Pearce; Tubbs et al.) congénitos expandidos que llevan a hidrocefalia (Pearce).

Esta cavidad es significativamente más frecuente en la mujer que en el hombre (Aldur et al.). En un estudio realizado de manera aleatoria al investigar la incidencia post mortem del cavum septum pellucidum en 500 cadáveres que oscilaban entre 14 y 89 años, se determinó entre otros, que esta cavidad es una anormalidad de estructuras de la línea mediana algunas veces asociadas con desórdenes y trastornos mentales (Filipovic et al.). Otro estudio de meta análisis de 1054 pacientes con esquizofrenia contra 886 voluntarios normales, demostró que la incidencia de dicho espectro era significativamente alta en personas que poseían un amplio cavum septum pellucidum (Trzesniak et al., 2011). El cavum septum pellucidum se encuentra en todos los neonatos y comienza a cerrarse poco antes del nacimiento en los nacidos a término y a menudo se observa en las imágenes cerebrales obtenidas por tomografía computarizada y resonancia magnética nuclear, sólo el 6\% llega a presentarse más allá de los 6 meses de edad (Afifi \& Bergman). En infantes de menos de 36 semanas de edad gestacional todos tienen cavum septum pellucidum y se piensa que esta cavidad permanece en solo el $36 \%$ de los infantes neonatos de término completo (Filipovic et $a l$.). Los niños que tienen un cavum septum pellucidum tan largo como $0,95 \mathrm{~cm}$ y tan profunda como $0,81 \mathrm{~cm}$ pueden tener una aberración en la maduración del desarrollo de estructuras de la línea mediana del cerebro (Bodensteiner \& Schaefer).

\section{MATERIAL Y MÉTODO}

Fueron disecados 42 cerebros de personas adultas, considerados normales desde la apariencia macroscópica. Luego de su extracción craneal, se fijaron en formalina al $10 \%$ por períodos variables de más de un mes, para posteriormente resecar las meninges y tomando como referencia la rodilla del cuerpo calloso por visualización a través de la fisura interhemisférica, se realizaron cortes coronales a nivel de esta región con el fin de identificar la presencia del cavum septum pelllucidum. Posterior a esta visualización en el sentido anteroposterior, se realizaron en los mismos cerebros, cortes horizontales a nivel de la comisura blanca anterior para apreciar la apariencia o no en el sentido ínfero-superior de dicho cavum septum pellucidum.

\section{RESULTADOS}

Se identificó de 42 cerebros de adultos, un cerebro de un individuo con un cavum septum pellucidum con las siguientes característica métricas: un ancho en su porción más anterior de $1,04 \mathrm{~cm}$-como referente se introdujo una sonda acanalada de $0,5 \mathrm{~cm}$ de diámetro (Fig. 1), una longi-

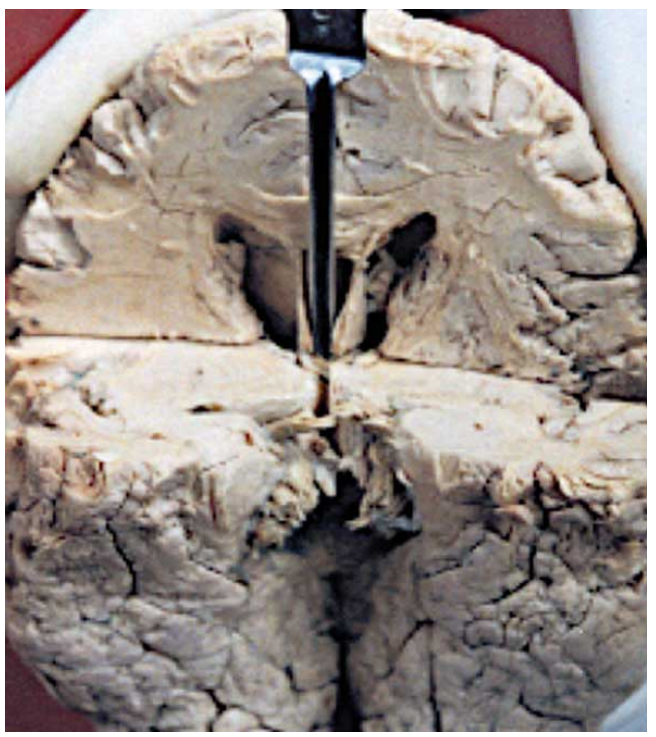

Fig, 1. Corte coronal y horizontal de un cerebro que muestra una sonda acanalada de $0,5 \mathrm{~cm}$ de diámetro, introducida en el cavum septum pellucidum, interpuesto entre los ventrículos laterales.

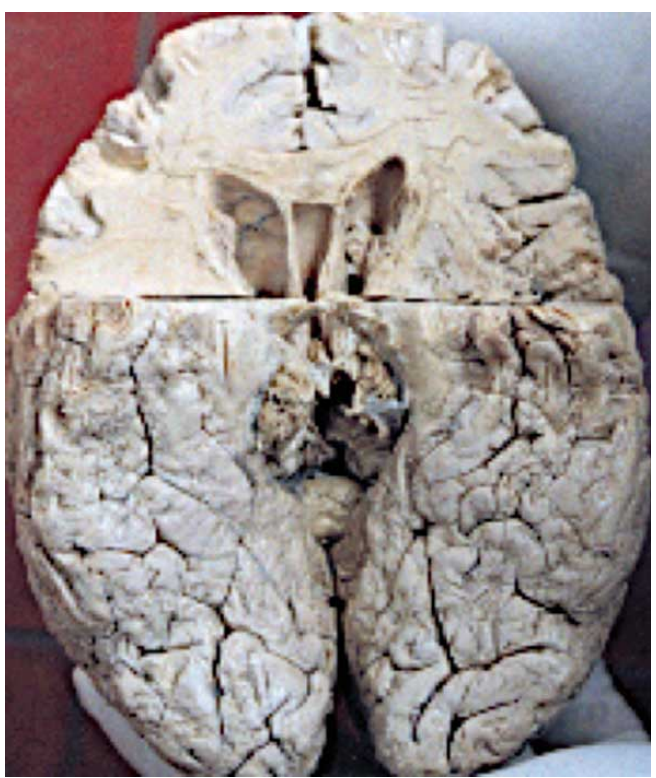

Fig, 2. Corte horizontal y coronal cerebral que muestra tres cavidades a nivel de la región frontal por detrás de la rodilla del cuerpo calloso: los ventrículos laterales y en posición intermedia el cavum septum pellucidum. 
tud de 2,1 cm que le permitía prolongarse hasta el tronco del cuerpo calloso. Su tamaño cualitativamente era aproximadamente el de un ventrículo lateral en su región frontal (Fig. 1 y 2) y las dos láminas claramente separadas.

\section{DISCUSIÓN}

Diversos autores han reportado que el cavum septum pellucidum es un hallazgo normal durante la vida intrauterina, pero no de forma usual como algo evidente en el adulto sano. La relación que se ha planteado de su existencia en pacientes que tienen trastornos conductuales como esquizofrenia (Grosmann \& Yousem; Trzesniak et al.), demencia pugilística, etc, hace suponer que este hallazgo se relacione con alteración conductual, puesto que este cerebro fue obtenido de un cadáver de un hospital psiquiátrico, además, de que en sus dimensiones, el referente ancho es evidentemente marcado con 1,04 cm, que supera extensamente el valor dado de 2 a $3 \mathrm{~mm}$ como normal, lo que proporcionalmente representa un aumento de 34 veces su ancho como valor mínimo y 52 veces como máximo. Desde el punto de vista cualitativo el grado de aumento del tamaño es tal, que da la apariencia de un ventrículo interpuesto entre los ventrículos laterales en la región de las astas ventriculares frontales (Figs. 1 y 2).

Debe considerarse además que los valores reales pueden ser mayores a los indicados, debido a que este cerebro fue fijado con formalina y se sabe que los valores de las estructuras fijadas, altera las dimensiones verdaderas (Saul et al., 1988) pues la formalina produce retracción de los tejidos.

\section{CONCLUSIÓN}

El cavum septum pellucidum es una cavidad que se manifiesta en la línea mediana del cerebro como componente natural durante el neurodesarrollo, pero la característica de gran tamaño que persiste o se genera posnatalmente, se asocia con alteraciones conductuales por lo que cabe suponer que este hallazgo neuroanatómico único, con medidas que sobrepasan entre 34 y 52 veces las medidas características de normalidad, corresponden a una persona con esquizofrenia al provenir de un centro psiquiátrico.

DUQUE PARRA, J. E. Cavum septum pellucidum: finding from neuroanatomical dissection. Int. J. Morphol., 30(4):1508-1511, 2012.

SUMMARY: A marked cavitation in the septum pellucidum level is reported, a great cavum septum pellucidum of an adult in the closed space generally recognized as the central nervous system, which is evident in itself, at certain stages of intrauterine life. This cavitation is usually absent in the healthy adult, but in some people with schizophrenia and pugilistic dementia, the cavity can be regarded and found relatively frequently. This finding was found randomly in one of forty-two adult brains, superficially valued from the gross anatomy as normal. This finding may be associated with behavioral disorders, taking in to account that the brain corresponded to a person from a psychiatric hospital.

KEY WORDS: Cavum septum pellucidum; Neuroanatomy; Dissection.

\section{REFERENCIAS BIBLIOGRÁFICAS}

Afifi, A. K. \& Bergman, R. A. Neuroanatomía funcional texto y atlas. México D. F., McGraw Hill Interamericana, 1999.

Aldur, M. M.; Celik, H. H.; Sargon, M. F.; Dagdeviren, A.; Aksit, M. D. \& Taner, D. Unreported anatomical variation of septum pellucidum. Clin. Anat., 10(4):245-9, 1997.

Bodensteiner, J. B. \& Schaefer, G. B. Wide cavum septum pellucidum: a marker of disturbed brain development. Pediatr. Neurol., 6(6):391-4, 1990.

Bogfdanoff, B. \& Natter, H. M. Incidence of cavum septum pellucidum in adults: a sign of boxer's encephalopathy. Neurology, 39(7):991-2, 1989.
Clemente, C. C. Anatomy of the human body. Baltimore, Williams \& Wilkins, 1985.

Duque Parra, J. E. Cavum septum pellucidum en el ser humano? Rev. Med. Cal., 15(3):119-26, 2001.

Duque Parra, J. E. Vasculatura del septum pellucidum humano. Biosalud, 2:63-7, 2002.

Duque Parra, J. E. Cavum del septo pelúcido y líquido cefalorraquídeo. Rev. Neurol., 38(4):400, 2004.

Filipovic, B.; Teofilovski-Parapid, G.; Pejkovic, B. Cavum septi pellucidi- Variation or abnormality? A post-mortem study. Braz. J. Morphol. Sci., 13:207-1, 1996. 
Grossman, R. I. \& Yousem, D. M. Neuroradiología. Madrid, Marbán Libros S. L., 2007.

Kiernan, J. A. El sistema nervioso humano un punto de vista anatómico. México, McGraw-Hill Interamericana, 2009.

Kuhlenbeck, H. The central nervous system of vertebrates. Vol III/ 2. Basel, Karger, 1973.

Martin, J. H. Neuroanatomía. Madrid, Prentice Hall, 1998.

Pearce, J. M. Some Observations on the Septum Pellucidum. Eur. Neurol., 59(6):332-4, 2008.

Ranson, S. W. \& Clark, S. L. The anatomy of the nervous system its development and function. Philadelphia, W. B. Saunders Company, 1959.

Saul, R. A.; Stevenson, R. E.; Rogers, R. C.; Skinner, S. A.; Prouty, L. A. \& Flannery, D. V. Growth references from conception to adulthood. Greenwood, Greenwood Genetic Center, 1988.

Tubbs, R. S.; Krishnamurthy, S.; Verma, K.; Shoja, M. M.; Loukas, M.; Mortazavi, M. M. \& Cohen-Gadol, A. A. Cavum velum interpositum, cavum septum pellucidum, and cavum vergae: a review. Childs. Nerv. Syst., 27(12):1927-30, 2011.

Testut, J. \& Latarjet, A. Tratado de Anatomía humana. Barcelona, Salvat Editores, 1978.

Trzesniak, C.; Oliveira, I. R.; Kempton, M. J.; Galvao de Almeida, A.; Chagas, H. N.; Ferrari, M. C.; Filho, A. S.; Zuardi, A. W.; Prado, D. A.; Busatto, G. F.; McGuire, P. K.; Hallak, J. E. \& Crippa, J. A. Are cavum septum pellucidum abnormalities more common in schizophrenia spectrum disorders? A systematic review and meta-analysis. Schizophr. Res., 125(1):1-12, 2011.

Zimmermann-Paiz, M. A \& Fang Sung, J. W. Hipoplasia del nervio óptico y displasia septo óptica. Arch. Argent. Pediatr., 107(6):542-56, 2009.

\author{
Dirección para Correspondencia: \\ Jorge Eduardo Duque Parra \\ Departamento de Ciencias Básicas \\ Programa de Medicina \\ Facultad de Ciencias para la Salud. \\ Universidad de Caldas \\ Manizales \\ COLOMBIA
}

Email: jorge.duque_p@ucaldas.edu.co

Recibido : 27-11-2011

Aceptado: 17-06-2012 